\title{
TRADUÇÃO E ADAPTAÇÃO CULTURAL DO WORC: UM QUESTIONÁRIO DE QUALIDADE DE VIDA PARA ALTERAÇÕES DO MANGUITO ROTADOR
}

\author{
Lopes AD ${ }^{1,2,3}$, Stadniky SP ${ }^{1,2,3}$, Masiero D ${ }^{1,3}$, Carrera EF ${ }^{2,3}$, Ciconelli RM ${ }^{4}$ e Griffin $S^{5}$ \\ ${ }^{1}$ Centro de Reabilitação, Lar Escola São Francisco, Escola Paulista de Medicina, Universidade Federal de São Paulo - \\ Unifesp, São Paulo, SP - Brasil \\ ${ }^{2}$ Grupo de Ombro e Cotovelo, Unifesp, São Paulo, SP - Brasil \\ ${ }^{3}$ Departamento de Ortopedia e Traumatologia, Unifesp, São Paulo, SP - Brasil \\ ${ }^{4}$ Disciplina de Reumatologia, Unifesp, São Paulo, SP - Brasil \\ ${ }^{5}$ Kirkley Research Group, Fowler Kennedy Sport Medicine Clinic, University of Western Ontario, London, Ontario - \\ Canada \\ Correspondência para: Andréa Diniz Lopes, Rua Dr. Diogo de Faria, 539 apto 25, Vila Clementino, CEP 04037-001, São \\ Paulo, SP - Brasil, e-mail: adinizlopes@uol.com.br
}

Recebido: 07/11/2005 - Aceito: 02/05/2006

\begin{abstract}
RESUMO
Objetivo: Realizar a tradução e a adaptação cultural do questionário WORC (The Western Ontario Rotator Cuff Index) para a língua portuguesa para ulterior validação no Brasil. Métodos: O protocolo aplicado consistiu em: 1) Preparação, 2) Tradução, 3) Tradução de volta à língua original (retro-tradução), 4) Interrogatório Cognitivo e 5) Relato de Informações. Ao serem concluídas as etapas de tradução e retro-tradução, as versões foram enviadas para os autores do WORC original, que as aprovaram para continuação do estudo. A versão em Português foi aplicada a 35 pacientes com disfunções do manguito rotador para verificar o nível de compreensão do instrumento. A idade média foi 57 anos (DP=13), 63\% eram do sexo feminino e 74\% tinham nível de escolaridade de primeiro grau incompleto. A versão brasileira final do WORC foi definida após se conseguir menos que 15\% de "não compreensão” em cada item. Para análise das variáveis, foi utilizada estatística descritiva. Resultados: Realizaram-se mudanças e substituições de termos e expressões para obter equivalência cultural do WORC. Alteraram-se também os termos “não compreendidos” pelos pacientes de acordo com as sugestões feitas por eles. Conclusão: Após a tradução e adaptação cultural do questionário, foi concluída a versão em Português do WORC que está em processo de validação para ser utilizada no Brasil.
\end{abstract}

Palavras-chave: qualidade de vida, questionários, tradução (produto), manguito rotador.

\section{ABSTRACT \\ Translation and Cultural Adaptation of WORC: a Quality-of-Life Questionnaire for Rotator Cuff Disorders}

Objective: To accomplish the translation of WORC (The Western Ontario Rotator Cuff Index) into Portuguese and its cultural adaptation, for future validation in Brazil. Method: The protocol applied consisted of: 1) Preparation; 2) Forward translation; 3) Back translation; 4) Cognitive Debriefing; and 5) Information report. After concluding the forward and back-translations, the versions were sent to the authors of the original questionnaire, who gave their approval for the study to be continued. The Portuguese version was applied to 35 patients with rotator cuff dysfunctions to verify the level of understanding of the instrument. The mean age of these patients was 57 years (SD = 13); $63 \%$ were female and $74 \%$ had not completed elementary school. The final Brazilian version of WORC was defined after achieving a "no comprehension" rate of less than $15 \%$ for each item. Descriptive statistics were used to analyze the variables. Results: Some terms and expressions were changed or replaced to obtain cultural equivalence for WORC. The terms that were incomprehensible to the patients were changed in accordance with their own suggestions. Conclusion: After the translation and cultural adaptation of the questionnaire, the final Portuguese version of WORC was concluded and it is now undergoing validation for use in Brazil.

Key words: quality of life, questionnaires, translation (product), rotator cuff. 


\section{INTRODUÇÃO}

Nos últimos anos têm ocorrido mudanças na abordagem das afecções musculoesqueléticas. No passado, avaliavamse as mudanças clínicas através do exame físico e exames complementares. Atualmente, desfechos como qualidade de vida têm sido enfatizados por possibilitarem a análise da situação de saúde e o impacto da doença na vida do indivíduo e por fornecer informações para avaliar efetividade de diferentes tratamentos. Para tanto foram desenvolvidas medidas de avaliação que abordam essa variável, já que o exame físico é um indicador insuficiente para avaliação de aspectos funcionais, sociais e emocionais ${ }^{1-4}$.

Com o aumento do interesse em se mensurar qualidade de vida, vários instrumentos ou questionários foram propostos ${ }^{4}$, sendo que a maioria deles foi desenvolvida na língua inglesa. Esses instrumentos podem ser usados tanto na prática clínica quanto em pesquisa. Entretanto, a multiplicidade dos mesmos dificulta a escolha apropriada frente a uma determinada população ${ }^{1}$.

Para utilização de medidas de avaliação em saúde desenvolvidas e utilizadas em outro idioma é necessário realizar a equivalência transcultural, sendo desnecessário criar e validar outro instrumento que avalie a condição de interesse. Esse processo consiste em realizar a tradução, adaptação cultural e validação do instrumento proposto. As etapas de tradução e adaptação cultural permitem ajustar o instrumento ao novo idioma, população, contexto e cultura. A fase de validação consiste em verificar se o novo instrumento manteve as características da versão original. As propriedades psicométricas mais comumente analisadas nessa fase são validade, reprodutibilidade e sensibilidade a mudanças. Todo esse processo é relevante para que o instrumento seja culturalmente aceito no país em questão e equivalente à versão

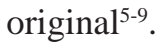

As disfunções do ombro são causas importantes de morbidade e incapacidade ${ }^{10}$. Das afecções do ombro, têmse as desordens do manguito rotador como as mais comuns ${ }^{1,11}$, sendo responsáveis por até $70 \%$ dos episódios de dor no ombro $^{10}$. Portanto, estudos têm sido propostos para avaliar o impacto dessa doença na qualidade de vida dos indivíduos e a efetividade de diferentes tratamentos ${ }^{12}$.

Os instrumentos de avaliação das afecções do ombro estão majoritariamente disponíveis em Inglês. Entre eles, o DASH (Disabilities of the Arm, Shoulder and Hand scale), o ASES (American Shoulder and Elbow Surgeons Standardized Shoulder Assessment Form), o SRQ (Shoulder Rating Questionnaire), o UCLA Shoulder Score e o SPADI (Shoulder Pain and Disability Index) $)^{13,14}$. Entretanto, nenhum instrumento específico para pacientes com alterações do manguito rotador havia sido desenvolvido seguindo um rigor metodológico adequado de validação $0^{15}$. Os instrumentos DASH ${ }^{16}$ (avalia sintomas e função nas afecções dos membros superiores) e Athletic Shoulder Outcome Rating Scale ${ }^{17}$ (escala específica para afecções do ombro em atletas) possuem versões traduzidas e validadas para o Português de acordo com os critérios recomendados ${ }^{5,6}$.

O WORC (The Western Ontario Rotator Cuff Index) é um questionário de qualidade de vida desenvolvido na língua inglesa. O estudo de suas propriedades psicométricas mostrou moderada a forte correlação com os instrumentos DASH, UCLA e ASES, enquanto na avaliação da reprodutibilidade, o índice de correlação intraclasse foi considerado excelente ${ }^{15}$. Foi criado e validado para ser aplicado a pacientes com alterações do manguito rotador, incluindo tanto a síndrome do impacto quanto as lesões e rupturas do manguito. Sendo um instrumento específico, engloba aspectos de qualidade de vida relevantes para essa doença. Contém 21 questões, abrangendo cinco domínios: 1) sintomas físicos, 2) trabalho, 3) esportes e recreação, 4) estilo de vida e 5) estado emocional ${ }^{15}$.

$\mathrm{O}$ formato de resposta às questões do WORC é através de Escala Visual Analógica (EVA). Todas as questões têm o mesmo valor ponderal. Portanto, cada item tem a possibilidade de ser pontuado de 0 a 100 na EVA e o resultado final pode variar de 0 a 2100. O total de 0 implica em nenhuma redução na qualidade de vida e 2100 é a pior pontuação. É possível obter o total de cada domínio separadamente. O resultado final pode ser convertido para percentagem de acordo com uma fórmula proposta pelos autores da versão original.

Apesar do WORC ter sido publicado recentemente, já existem traduções e validações em desenvolvimento em Francês e Alemão ${ }^{14}$.

O objetivo deste estudo foi realizar a tradução e adaptação cultural do questionário WORC para o Português do Brasil, com vistas a sua ulterior validação neste país.

\section{MÉTODOS}

\section{Participantes}

Participaram do estudo 35 pacientes, que tinham diagnóstico clínico de desordem do manguito rotador, de níveis escolar, socioeconômico e cultural variados e que estavam em tratamento fisioterapêutico. A idade média foi 57 anos (DP $=13$ anos) e 63\% eram do sexo feminino. Quanto ao nível de escolaridade, $74 \%$ tinham primeiro grau incompleto, $6 \%$ primeiro grau completo, $14 \%$ segundo grau completo e $6 \%$ terceiro grau completo.

Foram excluídos do trabalho indivíduos com alterações cognitivas, doenças neurológicas e reumáticas ou outras afecções específicas da articulação do ombro.

O estudo foi aprovado pelo Comitê de Ética em Pesquisa e foi obtida autorização dos autores do WORC original via correio eletrônico para utilização do mesmo.

\section{Procedimentos}

As traduções para o Português e o processo de adaptação cultural do WORC foram realizados de acordo com 
o protocolo específico de equivalência lingüística sugerido e enviado pelos autores da versão original do WORC. Esse protocolo segue os critérios definidos pelo MAPI Research Institute $^{18}$ e segundo Aquadro et al. ${ }^{19}$. Inclui cinco fases: preparação, tradução, tradução de volta à língua original (retrotradução), interrogatório cognitivo e relato das informações.

O processo de equivalência lingüística iniciou-se através do contato com os autores do WORC original para definições de seus conceitos e autorização para utilização do mesmo (fase de preparação).

O WORC foi traduzido inicialmente por dois tradutores juramentados independentes que tinham como língua mãe o Português. Sendo assim, obtiveram-se duas versões diferentes do questionário: "VA1" e "VA2”. Após essa etapa, foi realizado um encontro com ambos os tradutores e o pesquisador principal, os quais definiram uma versão consensual "VB" em Português.

A "VB" foi entregue a um terceiro tradutor juramentado, o qual tinha como língua mãe o Inglês, fluência na língua Portuguesa e que não conhecia o questionário original. O questionário foi então traduzido de volta para o Inglês (retrotradução). A versão original, a "VB" e a retro-tradução foram comparadas durante um encontro entre o último tradutor e o pesquisador principal, que estabeleceram uma versão consensual em Português "VC".

As traduções foram enviadas para os autores da versão original, que as analisaram e aprovaram de acordo com os critérios do MAPI Research Institute ${ }^{18}$ para manter a originalidade do questionário, reconhecê-lo como versão oficial da língua alvo e evitar a proliferação de versões desautorizadas.
Após ser analisada e aceita a "VC", foi realizado o teste de clareza, compreensão e aceitabilidade com cinco indivíduos. Essa fase foi denominada como primeira fase do Interrogatório Cognitivo.

O pesquisador principal leu em voz alta os itens do instrumento para esses indivíduos. Estes tinham que: 1) responder se compreenderam (sim ou não), 2) comentar o que entenderam de cada item lido e 3) sugerir alterações caso existisse algum item "não compreendido". De acordo com os resultados desse teste, a tradução foi modificada e atualizada, para estabelecimento da versão "VD”.

A versão "VD” foi então aplicada a outros 15 pacientes numa segunda fase do Interrogatório Cognitivo para refinar o teste de clareza e compreensão do instrumento, sendo que os itens com 15\% ou mais de "não compreensão" foram reformulados. Após essa etapa, o questionário foi aplicado a outros 15 pacientes com o mesmo propósito, numa terceira fase do Interrogatório Cognitivo. Finalmente, a versão brasileira do WORC foi definida.

Junto ao questionário, foram coletados alguns dados individuais como idade, sexo e grau de escolaridade. Ademais, cada fase do processo de equivalência lingüística foi documentada, detalhando os problemas encontrados e as soluções sugeridas e adotadas durante o processo.

A Estatística usada no estudo foi descritiva.

\section{RESULTADOS}

Na fase de tradução realizaram-se algumas alterações durante o encontro entre os dois tradutores e o pesquisador

Tabela 1. Modificações na fase de tradução.

Item do questionário-Termo

“VA1" e "VA2"

Modificação para versão consensual “VB”

\begin{tabular}{lll}
\hline 10- Push-ups & VA1- Flexões & Flexão de Braços \\
& VA2- Força & Áreas externas da casa \\
11- Yard & VA1 e VA2- Quintal & Braço não afetado \\
13- Uninvolved arm & VA1- Braço sadio & Braço machucado \\
& VA2- Braço não afetado & Carregar alguém no ombro ou brincar de “cavalinho” \\
13- Injured arm & VA1- Braço prejudicado & VA2- Braço machucado \\
17- "Roughhousing or horsing around” & VA1- Rolar no chão ou brincar de cavalinho & Deprimido, triste \\
& VA2- Brincar de rolar no chão ou de cavalinho
\end{tabular}

VA1: versão em Português referente ao primeiro tradutor. VA2: versão em Português referente ao segundo tradutor.VB: versão consensual em Português definida ao final da fase de tradução. 
principal. Foram discutidos todos os itens das versões "VA1" e "VA2” para formulação da versão consensual "VB” (Tabela 1).

No encontro entre o tradutor da fase de retro-tradução e o pesquisador principal foram avaliadas e comparadas a versão original, a "VB" e a retro-tradução para definir a "VC". Nessa etapa, foram feitas alterações em estruturas gramaticais de alguns itens para adquirir equivalência entre as palavras, entre os idiomas e adaptações de contexto cultural (Tabela 2).

Essas versões foram enviadas por correio eletrônico para os autores da versão original do WORC, que sugeriram algumas alterações (Tabela 3).

Os itens “não compreendidos” e alterados nas fases de interrogatório cognitivo estão incluídos nas Tabelas 4 e 5.
Após o resultado desse teste, foi definida a versão final brasileira do WORC.

\section{DISCUSSÃO}

Guillemin ${ }^{5}$ e Guillemim, Bombardier e Beaton ${ }^{6}$, propõem um procedimento padronizado para tradução e adaptação cultural de instrumentos. Esse procedimento tem sido seguido e citado em vários estudos e os critérios são reconhecidos internacionalmente. Porém, esse processo muitas vezes é de difícil realização dependendo da população estudada. A complexidade das etapas, a longa duração e alto custo são os principais pontos questionados ${ }^{20,21}$. O procedimento de tradução e adaptação cultural do WORC para o Português seguiu o protocolo sintetizado sugerido e enviado pelos autores

Tabela 2. Fase de retro-tradução. Modificações na "VB" para definição da "VC".

“VB”. Item do questionário -Termo

“VC”. Item do questionário -Termo

$\begin{array}{ll}\text { 6- Quanto desconforto você sente no pescoço por causa do seu ombro? } & \text { 6- Quanto desconforto você sente nos músculos do pescoço por causa } \\ & \text { do seu ombro? } \\ \text { 11- Passar o rastelo } & \text { 11- Varrer }\end{array}$ VB: Versão consensual em Português da fase de tradução. VC: Versão definida em Português após análise das versões original, da VB e da retro-tradução.

Tabela 3. Fase de retro-tradução. Sugestões dos autores do WORC original.

\begin{abstract}
5- How much popping, creaking do you feel in your - Popping: substituir por outro termo ou manter apenas creaking.
shoulder?

- Mudar o formato da frase para: "How much are you bothered by cliking, grinding or crunching in your shoulder?”
\end{abstract}

17- Carrying someone on your shoulder or playing Qualquer termo que signifique brincar/rolar no chão com qualquer pessoa.

"piggy back" with children.

21- Restless

Worried

Pontuaction

Scoring 
Tabela 4. Primeira fase do interrogatório cognitivo.

\begin{tabular}{ll}
\hline \multicolumn{1}{c}{ Item - Termo “não compreendido” } & Modificação sugerida pelos pacientes \\
\hline 1- Aguda & 1- Forte \\
4- Rigidez & 4- Rigidez, dureza \\
4- Período de inatividade & 4- Repouso \\
5- Estalido, rangido, crepitação & 5- Estala, range, crepita \\
7- Nível de desempenho físico & 7- Nível de condição física \\
9- Receio & 9- Medo \\
10- Exercícios vigorosos & 10- Exercícios pesados \\
14- Nível & 14- Altura \\
13- Compensar & 13- Substituir \\
13- Braço não afetado & 13- Braço bom \\
\hline
\end{tabular}

Tabela 5. Segunda e terceira fases do interrogatório cognitivo.

\begin{tabular}{|c|c|c|c|}
\hline Segunda Fase & & Terceira Fase & \\
\hline \multicolumn{4}{|l|}{ Item -Termo “não compreendido” } \\
\hline & Pacientes (\%) & Item -Termo “não compreendido” & Pacientes (\%) \\
\hline 1- dor aguda & 13 & 1- dor aguda & 13 \\
\hline 2- dor constante & 13 & 2- dor constante & 7 \\
\hline 4- rigidez, “dureza” & 60 & 3- fraqueza & 7 \\
\hline 5- crepita & 60 & 5- crepita & 7 \\
\hline 7- desempenho físico & 13 & 19- frustração & 7 \\
\hline 12- Acima do nível da cabeça & 7 & & \\
\hline
\end{tabular}

da versão original ${ }^{18,19}$. Todas as versões em desenvolvimento do WORC em outras línguas utilizam esse mesmo protocolo. Na tradução e adaptação cultural do WOMAC (Western Ontario and Mcmaster Universities) para a língua portuguesa, foram utilizados o processo tradicional de tradução e adaptação cultural e um processo simplificado. Nos seus resultados não foram encontradas diferenças importantes entre as formas e conteúdos das versões originadas pelo processo tradicional e pelo simplificado ${ }^{20}$.

Na fase de tradução do WORC para língua portuguesa foram observadas duas expressões pertencentes à questão 17 ("roughhousing” $e$ "horsing around”) que não possuíam equivalência idiomática no Brasil. Na versão consensual "VB” foi traduzida como "carregar alguém no ombro ou brincar 
de cavalinho”. Enviado à autora da versão original, esta sugeriu que fosse alterado para qualquer tipo de brincadeira pesada que sobrecarregue o ombro. Entretanto, ficou definido e aceito como "brincar, rolar no chão", mantendo o significado do contexto da questão.

Alguns itens do WORC não obtiveram equivalência conceitual ao serem traduzidos novamente para a língua original na fase de retro-tradução. Na questão 1, o termo “sharp pain”, foi traduzido como “dor aguda”. Na fase de interrogatório cognitivo, um dos pacientes sugeriu que o termo fosse trocado para “dor forte”. Entretanto, o termo forte, apesar de ser encontrado como sinônimo de agudo no dicionário, não pareceu adequado. Finalmente, foi mantido o termo “dor aguda”.

Na questão 5, a retro-tradução ficou como “How much popping, creaking do you feel in your shoulder?” (Quanto estalido, rangido e crepitação você sente no seu ombro?). Entretanto, a questão enfatizava o quanto o paciente se incomodava com estalido, rangido ou crepitação, incluindo não só os sintomas, mas também os sons. Portanto, na definição da "VC" o item foi modificado para "Quanto você se sente incomodado quando seu ombro estala, range ou crepita?”.

Alguns outros termos foram alterados no processo de definição da "VC". O termo "passar o rastelo" foi substituído por "varrer” para obter equivalência experimental. Dessa forma o contexto da questão não foi alterado e o ato de varrer é mais abrangente para a cultura do Brasil. Da mesma forma, na tradução e adaptação cultural do DASH para a língua portuguesa, foram substituídos os termos "jogar frisbee" ou "bater" (baseball) para "pescar” ou "jogar peteca” ${ }^{16}$. No processo de equivalência cultural do KDQOL-SF, o termo "passar aspirador de pó”, foi substituído por "varrer o chão" 4 .

Alguns termos foram modificados na fase de Interrogatório Cognitivo, como “estalido, rangido e crepitação”, que foram melhor compreendidos na forma verbal "estala, range e crepita”. Já "rigidez” necessitou da adição do termo "dureza” para facilitar o entendimento do item. Entretanto, foi modificado novamente na segunda fase de Interrogatório Cognitivo para "Quanto você sente seu ombro endurecido ou travado?”, já que os termos “rigidez” e “dureza” permaneceram incompreensíveis.

O questionário WORC foi aplicado nos pacientes e depois analisado e alterado para obtenção da adaptação cultural de acordo com as sugestões feitas por eles. Alguns autores sugerem que essa fase seja composta por um comitê de profissionais da saúde, para realizar uma revisão detalhada do questionário e substituir itens inapropriados para a cultura $^{8,21}$. Guillemin ${ }^{5}$ sugere que nessa fase exista tanto um comitê de revisão quanto a aplicação em pacientes (pré-teste), embora cite que não está claramente estabelecido na literatura se todas essas fases são essenciais. Apesar de existirem variações metodológicas para o processo de tradução e adaptação cultural de questionários de qualidade de vida, o processo básico padronizado deve ser mantido com as fases de tradução, retro-tradução e adaptação cultural ${ }^{21}$.

As respostas de cada questão do WORC têm o formato de escala visual analógica (EVA). Não houve dificuldade de compreensão para respondê-las de acordo com o significado de progressão do sintoma representado por ela, pois as instruções do questionário explicam claramente como utilizar esse tipo de escala. Durante a fase de adaptação cultural do WOMAC para a versão brasileira, foram comparadas as aplicações do questionário sob a forma de escala de Likert e com a EVA. Embora houvesse melhor compreensão utilizando a escala de Likert, diferenças significantes entre as duas não foram encontradas ${ }^{20}$. Na validação do WOMAC original, as respostas de cada questão também foram obtidas através das duas escalas. Os resultados mostraram que todos os domínios do questionário tinham reprodutibilidade adequada em ambas escalas e não foram encontradas diferenças estatisticamente significantes entre os dois tipos ${ }^{22}$. Segundo Guyatt, Bombardier e Tugwell ${ }^{23}$ não há evidências que uma seja melhor que a outra.

Com a utilização do WORC no presente estudo, não houve grandes dificuldades para a tradução e a adaptação cultural. O uso de critérios pré-estabelecidos, a troca de informações e a colaboração dos autores da versão original facilitaram o referido processo.

É importante que todas as etapas do processo de tradução, adaptação cultural e validação de questionários sejam relatadas para que seja possível escolher instrumentos apropriados e adequadamente testados. Do contrário, poderiam levar a resultados tendenciosos ou errados com falhas desde a escolha inapropriada de um questionário até a utilização de questionários sem valor científico ou a utilização inadequada dos mesmos ${ }^{4}$.

Após a definição da versão final do questionário para língua alvo, é importante a aplicação do mesmo numa amostra significativa para avaliação das propriedades psicométricas (validade, reprodutibilidade e sensibilidade a mudanças). Estas, avaliadas e aprovadas estatisticamente, irão determinar o quanto o questionário é útil para o seu propósito original ${ }^{24}$. A versão brasileira do WORC encontra-se em processo de avaliação das propriedades psicométricas, após a finalização do processo o questionário estará disponível para ser utilizado no Brasil.

\section{CONCLUSÃO}

Após a tradução e adaptação cultural do questionário, obteve-se a versão brasileira do WORC. 


\section{REFERÊNCIAS BIBLIOGRÁFICAS}

1. Skutek M, Fremerey RW, Zeichen J, Bosch U. Outcome analysis following open rotator cuff repair. Early effectiveness validated using four different shoulder assessment scales. Arch Orthop Trauma Surg 2000; 120 (7-8):432-36.

2. Berlim MT, Fleck MPA. Qualidade de vida: um novo conceito para pesquisa e prática em psiquiatria. Rev Bras Psiquiatr 2003; 25 (4):249-52.

3. Mendes MF, Balsimelli S, Stagehaus G, Tilbery CP. Validação de escala de determinação funcional da qualidade de vida na esclerose múltipla para a língua portuguesa. Arq Neuropsiquiatr 2004; 62 (1):108-13.

4. Duarte PS, Miyasaki MCOS, Ciconelli RM, Sesso R. Tradução e adaptação cultural do instrumento de avaliação de qualidade de vida para pacientes renais crônicos (KDQOL-SF). Rev Assoc Med Bras 2003; 49 (4):375-81.

5. Guillemin F. Cross-cultural adaptation and validation of health measures. Scand J Rheumatol 1995; 24 (2):61-3.

6. Guillemin F, Bombardier C, Beaton D. Cross-cultural adaptation of health-related quality of life measures: literature review and proposed guidelines. J Clin Epidemiol 1993; 46 (12):1417-32.

7. Cantarelli FB, Simões MFJ, Oliveira LM, Ferraz MB, Szejnfeld VL. Qualidade de vida em pacientes com fraturas por osteoporose: adaptação cultural, reprodutibilidade e validação do “Osteoporosis Assessment Questionnaire-OPAQ”. Rev Bras Reumatol 1999; 39 (1):9-18.

8. Herdman M, Rushby JF, Badia X. Equivalence and translation and adaptation of health-related quality of life questionnaires. Qual Life Res 1997; 6 (3):237-47.

9. Teixeira-Salmela LF, Magalhaes Lde C, Souza AC, Lima Mde C, Lima RC, Goulart F. Adaptation of the Nottingham Health Profile: a simple measure to assess quality of life. Cad Saude Publica 2004; 20 (4):905-14.

10. Ostor AJK, Richards CA, Prevost AT, Hazleman BL, Speed CA. Interrater reproducibility of clinical tests for rotator cuff lesions. Ann Rheum Dis 2004; 63 (10):1288-92.

11. Michener LA, McClure PW, Karduna AR. Anatomical and biomechanical mechanisms of subacromial impingement syndrome. Clin Biomech (Bristol, Avon) 2003; 18 (5):369-79.

12. Ejnisman B, Andreoli CV, Soares BGO, Fallopa F, Peccin MS, Abdalla RJ, et al. Interventions for tears of the rotator cuff in adults. Cochrane Database Syst Rev 2004; (1):CD002758.

13. Bot SDM, Terwee CB, Windt DAWM van der, Bouter LM, Dekker J, Vet HCW de. Clinimetric evaluation of shoulder disability questionnaires: a systematic review of the literature. Ann Rheum Dis 2004; 63 (4):335-41.
14. Kirkley A, Griffin S, Dainty K. Scoring systems for the functional assessment of the shoulder. Arthroscopy 2003; 19 (10):110920.

15. Kirkley A, Alvarez C, Griffin S. The development and evaluation of a disease-specific quality-of-life questionnaire for disorders of the rotator cuff: The Western Ontario Rotator Cuff Index. Clin J Sport Med 2003;13 (2):84-92.

16. Orfale AG, Araújo PMP, Ferraz MB, Natour J. Translation into Brazilian Portuguese, cultural adaptation and evaluation of the reliability of the Disabilities of the arm, shoulder and hand questionnaire. Braz J Med Biol Res 2005; 38 (2):293-302.

17. Leme L. Tradução, Adaptação e Validação da Escala "Athletic Shoulder Outcome Rating Scale” para Língua Portuguesa [dissertação]. São Paulo: Universidade Federal de São Paulo, Escola Paulista de Medicina; 2005.

18. Acquadro C, Conway K, Girourdet C, Mear I. Linguistic Validation Manual for Patient Reported Outcomes(PRO) Instruments.Lyon (France): MAPI Research Trust; 2004. Available from:URL: http://www.mapi-research.fr/i_02_manu.htm.

19. Aquadro C, Jambon B, Ellis D, Marquis P. Language and Translation Issues. In: Spilker B, ed. Quality of Life and Pharmacoeconomics in Clinical Trials. Philadelphia: LippincottRaven; 1996. P.575-85.

20. Fernandes MI. Tradução e validação do questionário de qualidade de vida específico para osteoartrose WOMAC (Western Ontario McMaster Universities) para a língua portuguesa. [dissertação]. São Paulo: Universidade Federal de São Paulo, Escola Paulista de Medicina; 2003. Available from: URL:http:// www.biblioteca.epm.br/.

21. Falcão DM, Ciconelli RM, Ferraz MB. Translation and cultural adaptation of quality of life questionnaire: an evaluation of methodology. J Rheumatol 2003; 30 (2):379-85.

22. Bellamy N, Buchanan WW, Goldsmith CH, Campbell J, Stitt LW. Validation study of WOMAC: a health status instrument for measuring clinically important patient relevant outcomes to antirheumatic drug therapy in patients with osteoartritis of the hip or knee. J Rheumatol 1988; 15 (12):1833-40.

23. Guyatt GH, Bombardier C, Tugwell PX. Measuring diseasespecific quality of life in clinical trials. CMAJ 1986; 134 (8):889-95.

24. Ebrahim S. Clinical and public health perspectives and applications of health-related quality of life measurement. Soc Sci Med 1995; 41 (10):1383-94. 